\title{
Predictive role of Natural Anticoagulants in Prognosis of Chronic Liver Disease in Pediatric Age Group
}

Sana Ajmal, ${ }^{1}$ Hina Sehar, ${ }^{1}$ Saleha Zafar, ${ }^{2}$ Saima Farhan, ${ }^{1}$ Nisar Ahmad, ${ }^{1}$ Muhammad Atif Riza ${ }^{1}$

\begin{abstract}
Background: In asymptomatic peadiatric patients, cirrhosis is considered to be compensated, with or without gastroesophageal varices, it may be decompensated. Both Antithrombin III, Protein C and D dimers are sensitive markers of liver disease.

Objective: To determine the frequency of compensated and decompensated cirrhosis in cirrhotic peadiatric patients and compare the status of protein C, Antithrombin 111 deficiency and D-dimer in both groups.

Methodology: In this cross sectional study, 80 peadiatric patients suspected to have cirrhosis were included, from November, 2015 to April, 2016, by non-probability convenience sampling. Variables included were; compensated and decompensated chronic liver disease, Antithrombin III, D dimer and Protein C, as natural anticoagulents. Data was analyzed by SPSS 13.

Results: Out of 80 patients, $70(87.5 \%)$ were in the compensated phase of cirrhosis. Mean Antithrombin III was significantly low in patients with decompensated cirrhosis $(50.0 \pm 4.05 \%)$ when compared with patients with compensated liver disease $(84.52 \pm 15.36 \%)$. $(\mathrm{p}=0.000)$ Mean Protein $\mathrm{C}$ level exhibited also significant reduction in the decompensated patient groups $(40 \pm 4.7 \%)$ when compared with the compensated group of patients $(77.92 \pm 6.67 \%)$. $(\mathrm{p}=0.000)$ The mean D-dimer levels showed significantly higher levels only in patients with signs of decompensated cirrhosis $(840 \pm 11.85 \mu \mathrm{g} / \mathrm{L})$ when compared with compensated disease $(629 \pm 6.29) .(\mathrm{p}=0.000)$

Conclusion: Our study concluded that the natural anticoagulants Antithrombin III, Protein C and D dimer, reflect hepatocellular impairment in both compensated as well as decompensated peadiatric patients of cirrhosis. However, these are markedly decreased in decompensated disease except for D dimer.
\end{abstract}

Key words: Cirrhosis, Anti-thrombin III, Protein C, Protein S, D-dimer.

Article Citation: Ajmal S, Sehar H, Zafar S,Farhan S, Ahmad N, Riza MA. Predictive role of Natural Anticoagulants in Prognosis of Chronic Liver Disease in Pediatric Age Group. JSZMC 2020;11(1):28-33

\section{Introduction}

Cirrhosisis the outcome of damage to liver tissue and scarring of the liver (fibrosis, which is nodular regeneration) causing progressive decline in liver function, excessive fluid in abdomen (ascites), bleeding disorders (known as coagulopathy), raised pressure in the blood vessels (portal hypertension), and brain function disorders (which is hepatic encephalopathy). ${ }^{1}$ Chronic liver disease particularly in the end stage is characterized by clinical bleeding and decreased level of most procoagulant factors. In adition to that, there is decreased level of naturally occurring anticoagulants like antithrombin III and protein C. In physiological conditions the coagulation system is balanced by these opposing drivers. ${ }^{2}$

The liver plays a central role in blood coagulation process because it is the site of synthesis of most of the coagulation factors and their inhibitors. ${ }^{1,2}$ Chronic liver disease is commonly associated with complex haemostatic defects that include impaired synthesis of clotting factors, ${ }^{3,4,5,6}$ and coagulation inhibitors, ${ }^{7,89}$ abnormalities of fibrinolytic activitiy, ${ }^{10}$ disseminated intravascular coagulation and platelet defects. ${ }^{11}$ Past studies showed decreased circulating levels of natural anticoagulants in those with chronic liver disease, as well as acute liver failure, as a result of impaired liver synthetic function. ${ }^{12,13}$ Studies of the natural history of cirrhosis have found that the disease tends to present with a silent clinical course, followed by the onset of liver dysfunction and portal hypertension. The most important predictor of decompensation is the increase in hepatic venous pressure gradient (HVPG), which is seldom measured routinely in children and adolescents. In clinical practice, mortality risk is generally

1. The Children's Hospital and The Institute of Child Health, Lahore, Pakistan.

2. Department of Pathology, Quaid e Azam Medical College, Bahawalpur, Pakistan.

Correspondence: Dr. Sana Ajmal, Children Hospital and The Institute of Child Health Lahore, Pakistan.

Received: 20-02-2020 Reviewed: 05-03-2020 
estimated on the basis of albuminemia, MELD (Model for End-Stage Liver Disease)/PELD (Pediatric End-Stage Liver Disease) / Child Pugh Turcotte scores and body mass index. Advances in diagnostic and treatment technology, especially liver transplant surgery, have contributed significantly to the management of these cases. Currently, the majority of children diagnosed with cirrhosis in the first years of life can grow, develop and reach adulthood. ${ }^{11-13}$ Natural pattern of cirrhosisis based on an asymptomatic phase known as compensated cirrhosis, followed by the progression of complications from portal hypertension and liver dysfunction, called decompensated cirrhosis. The transition has been estimated to occur at a rate of 5\%-7\% per year. In recent years this process has been proposed as a series of critical steps that if unchecked, culminate in hepatic decompensation. ${ }^{14}$

The studies that provide more data on the natural history of cirrhosis are related to the evolution of chronic hepatitis by HBV and HCV. These are based on prospective, retrospective and cross sectional studies, but are conditioned by factors that make difficult to establish absolute evidence on the natural history of the disease. ${ }^{14,15}$ Of those patients with $\mathrm{HCV}, 50 \%$ usually develop chronic liver disease including cirrhosis and liver cancer. It is predicted that $15 \%$ of chronically infected persons develop liver cirrhosis within 20 years. However, there are individual differences. ${ }^{16-18}$ Currently it is known that $33 \%$ of patients develop cirrhosis in less than 20 years, while another $31 \%$ will need many more years in order to develop the same damage. Most patients are asymptomatic or have nonspecific symptoms until decompensation occurs. Causes of cirrhosis are biliary atresia, choledochal cysts, viral hepatitis, alpha-1antitrypsin deficiency, wilson's disease, cystic fibrosis, fatty liver disease, autoimmune diseases and mitochondrial diseases. ${ }^{19,20}$ The objective of this study was to determine the frequency of compensated and decompensated cirrhosis in cirrhotic peadiatric patients and compare the status of protein C, Antithrombin 111 deficiency and D-dimer in both groups.

\section{Methodology}

In this cross sectional study, a total of 80 consecutive children suspected to have cirrhosis fulfilling inclusion and exclusion criteria, were selected during six month period i.e from November, 2015 to April, 2016 from Department of Haematology and Transfusion at The Children's Hospital and The Institute of Child Health, Lahore after taking informed consent from parents. Potential confounders were controlled by strictly following inclusion and exclusion criteria. Peadiatric patients of 5 years to 15 years of age of either sex suspected to have cirrhosis, a history documenting anorexia, fever, vomiting, abdominal pain, darkening of the urine, mostly after ingestion of crustaceans or shellfish of dubious provenance, should lead to the impression of hepatitis A virus infection (HAV). Along with HBV and HCV, HAV infection also is the likely etiology of liver disease and on physical examination with plethora, hepatomegaly, jaundice were included in this study while patient presented with congenital antithrombin III and protein $\mathrm{C}$ deficiency on medical record, patients of chronic liver diseases, comorbid conditions like diabetes mellitus (fasting sugar $\geq 126 \mathrm{mg} / \mathrm{dl}$ on two occasion), renal failure associated with protienurea (nephritic syndrome $\geq 3.5 \mathrm{~g} / \mathrm{dl}$ ), RFT (creatinine $\geq 1.2 \mathrm{~m} / \mathrm{dl}$, urea $\geq 50 \mathrm{mg} / \mathrm{dl}$ ), hyperlipidemia (lipid profile, cholesterol $\geq 200 \mathrm{md} / \mathrm{dl}, \mathrm{TG} \geq 150 \mathrm{md} / \mathrm{dl}$ ), hepatosteatosis. Patient with the history of drug that interfere with antithrombin III and protein C and S deficiency and patients with the raised D-dimer due to other causes such as DIC (FDP $\geq 200 \mathrm{mg} / \mathrm{dl}$ ), sepsis (CRP, blood culture), sickle cell crisis (Hb electrophoresis), pneumonia (chest $\mathrm{x}$-ray, blood culture) were excluded from the study.

Cirrhosis was diagnosed in these patients on the basis of history, physical examination and nonspecific laboratory test like liver enzymes and Ultrasound abdomen for hepatomegaly and was diagnosed on liver biopsy showing fibrosis and nodular transformation. While according to child pugh classification, class A point (5-6), class B (7-9) is considered as compensated cirrhosis and class $\mathrm{C}$ point $10-15$ is considered as decompensated cirrhosis. For predictive role of natural anticoagulants in prognosis of chronic liver disease in pediatric age group.

Antithrombin III and Protein C: Collected in APTT vial was run on Stago STA compact while D-dimer: Collected in APTT vial was run manually. Antithrombin III deficiency, protein $\mathrm{C}$ deficiency, raised D-dimer as according to operational definitions are the outcome 
variables. Ethical approval was sought from ethical committee of hospital. All data entered in computer software SPSS version 20. Mean \pm S.D was applied to express the value of the variables in compensated and decompensated cirrhosis. Quantitative variables like protein $\mathrm{C}$, antithrombin III and D-dimer level in patients of cirhhosis was expressed as percentages and frequencies. Chi square test applied to analyze significant differences between qualitative variables like antithrombin III, protein C deficiency and raised d-dimer in compensated and decompensated cirrhosis. Data was analyzed by SPSS version 13.

\section{Results}

Out of 80 patients, 70 patients $(87.5 \%)$ were in the compensated phase of cirrhosis while $10(12.5 \%)$ of the patients were in the decompensated phase of cirrhosis. The frequency distribution of compensated and decompensated patients is shown in table I. significantly higher levels only in patients with signs of decompensated cirrhosis $(840 \pm 11.85 \mu \mathrm{g} / \mathrm{L})$ when compared with compensated disease $(629 \pm 6.29 \mu \mathrm{g} / \mathrm{L})$. (Table I) Data was stratified for the value of antithrombin III and 26 (72.2\%) compensated patients showed antithrombin III less than $80 \%$ while $44(100 \%)$ compensated patients were with antithrombin III between 80 to $120 \%$. In the category of decompensation all $10(27.8 \%)$ patient showed antithrombin III less than $80 \%$ with $\mathrm{P}$-value $=0.000$. In case of protein C, $9(47.4 \%)$ compensated patients showed protein $\mathrm{C}$, less than $70 \%$ while $10(52.6 \%)$ compensated patients were with protein C, 70 to $130 \%$, while in the category of decompensated group $10(52.6 \%)$ patient showed protein $\mathrm{C}$ less than $70 \%$ with P value of 0.000 .

Table I: Anticoagulant level in children with Chronic Liver Disease in compensated versus decompensated stage. $(n=80)$

\begin{tabular}{|c|c|c|c|}
\hline Variable & $\begin{array}{l}\text { Compensated Chronic } \\
\text { Liver Disease }\end{array}$ & $\begin{array}{c}\text { Decompensated Chronic } \\
\text { Liver Disease }\end{array}$ & P value \\
\hline Stage & $70(87.5 \%)$ & $10(12.5 \%)$ & \\
\hline Antithrombin III & $84.52 \pm 15.36$ & $50.0 \pm 4.05$ & \\
\hline Protein C & $77.92 \pm 6.67$ & $40.00 \pm 4.7$ & \\
\hline D-dimer & $629.6 \pm 6.29$ & $840 \pm 11.85$ & \\
\hline \multicolumn{3}{|c|}{ Stratification of Antithrombin III level in compensated and decompensated $\quad$ patients } & \multirow{4}{*}{0.000} \\
\hline Less than $80 \%(n=36)$ & $26(72.2 \%)$ & $10(27.8 \%)$ & \\
\hline 80 to $120 \%(n=44)$ & $44(100 \%)$ & $0(0.0 \%)$ & \\
\hline Total $(\mathbf{n}=\mathbf{8 0})$ & $70(87.5 \%)$ & $10(12.5 \%)$ & \\
\hline \multicolumn{3}{|c|}{ Stratification of Protein $\mathrm{C}$ level in compensated and decompensated patients } & \multirow{4}{*}{0.000} \\
\hline less than $70 \%(n=19)$ & $9(47.4 \%)$ & $10(52.6 \%)$ & \\
\hline 70 to $130 \%(n=61)$ & $61(100 \%)$ & $0(0.0 \%)$ & \\
\hline Total $(\mathbf{n}=\mathbf{8 0})$ & $70(87.5 \%)$ & $10(12.5 \%)$ & \\
\hline \multicolumn{3}{|c|}{ Stratification of D-dimer level in compensated and decompensated patients } & \multirow{4}{*}{0.000} \\
\hline less than $639 \mathrm{ng} / \mathrm{m}$ & $59(100 \%)$ & $0(0.0 \%)$ & \\
\hline more than $639 \mathrm{ng} / \mathrm{m}$ & $11(52.4 \%)$ & $10(47.6 \%)$ & \\
\hline Total $(n=80)$ & $70(87.5 \%)$ & $10(12.5 \%)$ & \\
\hline
\end{tabular}

The mean level of AT III showed significant reduction in patients with decompensated cirrhosis $(50.0 \pm 4.05 \%)$ when compared with patients with compensated liver disease $(84.52 \pm$ $15.36 \%$ ). At the same time the mean PC level exhibited a more significant reduction in the decompensated patient groups $(40 \pm 4.7 \%)$ when compared with the compensated group of patients (77.92 $\pm 6.67 \%)$. The mean D-dimer levels showed
In case of D-dimer, 59 (100.0\%) compensated patients showed D-dimer less than $639 \mathrm{ng} / \mathrm{l}$ while there is no patient in this group showed less than $639 \mathrm{ng} / \mathrm{l}$ while $11(52.4 \%)$ compensated patients were with D-dimer more than $639 \mathrm{ng} / 1$ while in the category of decompensation $10(47.6 \%)$ patient showed D-dimer more than $639 \mathrm{ng} / \mathrm{l}$ with $\mathrm{P}$-value of 0.000 . 


\section{Discussion}

It is well-known that chronic liver disease is characterized by variable haemostatic defects that involve primary haemostasis, fibrinolysis and coagulation. ${ }^{13}$ Coagulation indices due to their relationship to liver synthetic function are well defined as prognostic markers in a range of settings in both acute/chronic liver disease. ${ }^{14,15}$ Previous studies have shown in patients with chronic liver disease a marked decrease in liver synthesis of coagulation factors as well as inhibitors. ${ }^{18}$ Recently, anticoagulation proteins were approved not only to reflect hepatocyte impairment but also to have predictive value in chronic liver disease. $^{15}$

In the current report, the plasma levels of natural anticoagulants Antithrombin III and Protein C, exhibited significant reduction in patients with decompensated as compared to compensated cirrhosis. This is in line with previous studies, which reported low levels of natural anticoagulants not only in chronic liver disease but also in acute hepatitis. ${ }^{16}$ Natural anticoagulants are intimately related to liver function and Antithrombin III is only synthesized by liver tissue. ${ }^{16,17}$ Acute or chronic liver diseases may decrease the concentration of Antithrombin III. Several studies have investigated the reduction of Antithrombin III in chronic liver disease. ${ }^{18,19}$ The plasma concentration of Antithrombin III is too low in cirrhosis, as reported in a previous study, ${ }^{20}$ and concluded that altered plasma concentration of Antithrombin III in cirrhosis was due to reduced transcapillary flux ratios in his sample. It is clear that damage to hepatic tissue, particularly damage to the endothelium, triggers the included inflammation and upsetting of the physiological anticoagulant mechanism. ${ }^{21}$ Our data shows that patients with chronic hepatitis tend to have cirrhosis leading to decreased levels of Antithrombin III. We have suggested Antithrombin III as a marker for fibrosis, but this hypothesis should be tested in future studies. The structure behind the diminution of Antithrombin III in liver disease are complex, but inadequate hepatic synthesis, an altered trans capillary flux ratio, and a low diffuseness DIC may be involved in the process. Another recent published investigation documented Protein $\mathrm{C}$ as a potential predictor of hepatic fibrosis in chronic liver disease. These findings, including the present one, confirm that levels of Protein $\mathrm{C}$ are sensitive markers of hepatocyte impairment. Antithrombin III was shown to be a good marker of liver cell synthetic function in cirrhosis. ${ }^{22}$

In the present study we found high D-dimer levels in patients with decompensated liver cirrhosis while in compensated patients no significant increase of Ddimer levels were observed. Plasma levels of fragment D-dimer represent an accurate marker of fibrinolytic activity and increased fibrinolysis is a common finding in advanced liver disease. In liver cirrhosis pathogenesis of hyperfibrinolysis in liver is not yet clearly known. Having found high D-dimer levels in the blood and in the ascitic fluid, Agarwal et al suggested ascites among the possible causes of increased fibrinolysis in patients with liver cirrhosis. ${ }^{22}$ Our finding is in agreement with that of Agarwal et al who reported increased plasma Ddimer values in $93 \%$ of patients with cirrhosis and ascites. Another recent study confirmed the association between circulating high D-dimer levels and the presence of ascites found in cirrhotic patients. $^{23}$

On the basis of these findings it can be shown that in liver cirrhosis ascites counts to the main factors to be associated with increased fibrinolytic activity. We may conclude that high D-dimer levels in cirrhotics is a strong indicator of decompensation. The underlying mechanism for these observation remains to be clarified. Increased levels of D-dimer has been detected in ascitic fluid as well, suggesting that ascites reabsorption into systemic circulation contributes to hyperfibrinolytic state in patients with advanced liver disease. ${ }^{22,24}$ Piscaglia et al proposed that the association between high circulating Ddimer levels and ascites might be due only to advanced liver impairment in decompensation. ${ }^{25}$

The levels of coagulation factors and natural anticoagulant proteins synthesized in the liver are valuable in diagnosis and treatment, as they reflect the degree of hepatocyte damage. In this study, the levels of protein inhibitors and fibrin degradation products were measured in patients with parenchymal damage to investigate whether they can be used as markers of parenchymal damage.

In the present study, D-dimer, a marker of fibrin degradation products, was found to be elevated with increasing severity of hepatocyte damage. Since D- 
dimer was the only parameter that differed significantly between Child A and Child B patients, D-dimer levels may be considered as an important sign of decompensation in cirrhotic patients. Antithrombin III may be considered as an early marker of hepatocellular damage. In addition, D-dimer may be a marker of decompensation in addition to Protein $\mathrm{C}$ and Antithrombin III.

\section{Conclusion}

On the basis of our results we concluded that natural anticoagulants Antithrombin III and Protein $\mathrm{C}$ shows hepatocellular impairment in compensated as well as decompensated patients of cirrhosis. This is also supported that in decompensation there is marked decrease in these anticoagulant proteins. Our study further shows that patients with high D-dimer levels require careful monitoring since this observation is associated with decompensated liver disease. Whether these parameters could be used in the clinical assessment of liver impairment need to be confirmed by future larger studies

Authors Contribution: MG: Conception of work and Design of Work. SAR: Drafting and Revising. MFM: Acquisition \& analysis SG: Analysis of data and drafting.

All the authors gave final approval for publication and agreed to be accountable for all aspect of work.

\section{Conflict of Interest: None Sources of Funding: Self}

\section{References}

1. Mammen EF. Coagulation abnormalities in liver disease. Haematol Oncol Clin North Am. 1992; 6: 1247-1257.

2. McCormick PA, Murphy KM. Splenomegaly, hypersplenism and coagulation abnormalities in liver disease. Bailliere's Clin Gastroentrol 2000; 14: 10091031.

3. Tacke F, Fiedler K, von Depka M. Clinlical and prognostic role of plasma coagulation factor XIII activity for bleeding disorders and 6-year survival in patients with chronic liver disease. Liver Int 2006; 26: 173-181.

4. Rodríguez-Iñigo E, Bartolomé J, Quiroga JA, Hedner U, Suárez A, Tomás JF. Expression of factor VII in the liver of patients with liver disease: correlations with the disease severity and impairment in the hemostasis. Blood Coagul Fibrinolysis. 2001; 12: 193-199.
5. Kerr R. New insights into haemostasis in liver failure. Blood Coagul Fibrinolysis.2003; 12: 43-45.

6. Hollstelle MJ, Geertzen HG, Straatsburg IH, Van Gulik TM, Van Mourik JA. Factor VIII expression in liver disease. Thromb Haemost 2004; 91: 267-275.

7. De Caterina M, Tarantino G, Farina C, Arena A, di Maro G, Esposito P, Scopacasa F. Haemostasis unbalance in Pughscored liver cirrhosis: characteristic changes of plasma levels of protein C versus preotein S. Haemostasis 1993; 23:229-235.

8. Kujovich JL. Haemostatic defects in end stage liver disease. Crit Care Clin 2005; 21: 563-587.

9. Tripodi A, Mannucci PM. Abnormalities of hemostasis in chronic liver disease: reappraisal of their clinical significance and need for clinical and laboratory research. J Hepatol 2007 Apr; 46(4): 727-733.

10. Al Ghumlas AK, Abdel Gader AG, Al Faleh FZ. Haemostatic abnormalities in liver disease: could some haemostatic tests be useful as liver function tests? Blood Coagulation and Fibrinolysis 2005; 16:329-335.

11. O'Grady JG, Langly PG, Isola LM, Aledort LM, Wiliams R. Coagulopathy of fulminant hepatic failure. Semin Liver Dis. 1986;6:159-163.

12. Garcia-Tsao G, Friedman S, Iredale J, Pinzani M. Now there are many where before there was one: In search of a pathophysiological classification of cirrhosis. Hepatology 2010; 51: 1445-1449.

13. Tripodi A. Hemostasis abnormalities in liver cirrhosis: myth or reality? Pol Arch Med 2008 Jul-Aug; 118(7-8): 445-8.

14. Polson J, Lee WM. American Association for the Study of Liver Disease. AASLD position paper: the management of acute liver failure. Hepatology 2005 May; 41(5): 11791197.

15. Schepke M, Roth F, Fimmers R, Brensing KA, Sudhop T, Schild HH, Sauerbruch T. Comparison of MELD, ChildPugh, and Emory model for the prediction of survival in patients undergoing transjugular intrahepatic portosystemicshunting.Am J Gastroenterol. 2003 May; 98(5): 1167-1174.

16. Tripodi A, Mannucci PM. Abnormalities of hemostasis in chronic liver disease: reappraisal of their clinical significance and need for clinical and laboratory research. J Hepatol. 2007 Apr; 46(4): 727-733.

17. Abdo AA, Sanai FM, Azzam N, Al Sawat K, Al Dukhayil M, Al Ghumlas A, et al. Natural anticoagulants can be useful predictors of severity in chronic liver disease. Blood Coagul Fibrinolysis. 2010;21:122-127.

18. Cong YL, Wei YX, Zhang LW, Yin ZJ, Bai J. The relationship between hemostatic changes in liver cirrhosis patients with different degrees of liver lesions in reference to Child-Pugh scores. Zhonghua GanZang Bing ZaZhi 2005; 13(1):31-4.

19. Kerr R, Newsome P, Germain L. Effects of acute liver injury on blood coagulation. J Thromb Haemost 2003; 1(4): 754-9.

20. Romero Gomez M, Suarez Garcia E, Lopez Lacomba D, Marchante I, Grande L, Castro Fernandez M. Antiphospholipid antibodies are related to portal vein thrombosis in patients with liver cirrhosis. J Clin Gastroenterol 2000; 31(3): 237-40. 
21. Carey MJ, Rodgers GM. Disseminated intravascular coagulation: clinical and laboratory aspects. Am J Hematol 1998; 59(1): 65-73.

22. Agarwal S, Joyner KA Jr, Swaim MW. Ascites fluid as a possible origin for hyperfibrinolysis in advanced liver disease. Am J Gastroenterol 2000; 95: 3218-3224

23. Spadaro A, Tortorella V, Morace C, Fortiguerra A, Composto P, Bonfiglio $\mathrm{C}$ et al. High circulating Ddimers are associated with ascites and hepatocellular carcinoma in liver cirrhosis. World J Gastroenterol 2008; 14: 1549-1552.
24. Toschi V, Rocchini GM, Motta A, Fiorini GF, Cimminiello C, Violi F. The hyperfibrinolytic state of liver cirrhosis: possible pathogenetic role of ascites. Biomed Pharmacother.1993; 47:345-352.

25. Piscaglia F, Donati G, Giannini R, Bolondi L. Liver cirrhosis, ascites, and hyperfibrinolysis. Am J Gastroenterol 2001; 96:3222-7. 